DOI 10. 18307/2017. 0525

(C) 2017 by Journal of Lake Sciences

\title{
基于矩阵模型进行抗荧光干扰的蓝藻原位检测方法
}

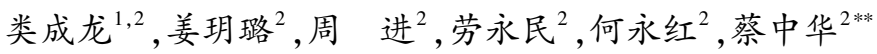 \\ (1: 清华大学生命学院, 北京 100084) \\ (2:清华大学深圳研究生院, 深圳 518055$)$
}

\begin{abstract}
摘 要: 藻蓝蛋白与叶绿素 $\mathrm{a}$ 作为特征色素常用于表征蓝藻的生物量. 藻蓝蛋白与叶绿素 $\mathrm{a}$ 通常用苂光光谱或原位苂光 强度检测,但是在实际水体中藻蓝蛋白与叶绿素 $\mathrm{a}$ 的苂光效应相互干扰会影响测量精度,极大限制了该方法的应用. 本文 根据朗伯比尔定律, 利用二阶矩阵模型设计了一套在苂光检测过程中 “激发光-发射光”优化抗干扰的波长选择方法, 并 利用多元线性回归分析建立了两种色素浓度与荧光强度之间的多元校正线性模型, 实验验证了所䇥选蓝藻苂光分析法 中特定激发光和发射光波长的有效性. 本研究实现了两种色素检测过程中的成功解舶, 可在藻蓝蛋白的原位检测中排除 水体中藻蓝蛋白与叶绿素 $\mathrm{a}$ 之间的相互干扰, 提高藻蓝蛋白和叶绿素 $\mathrm{a}$ 的原位检测精度, 为开发便携式蓝藻检测传感器 时的波长选择提供理论基础.
\end{abstract}

关键词: 藻蓝蛋白; 叶绿素 $\mathrm{a}$; 苂光检测; 解耦; 抗苂光干扰

\section{Anti-interferance in-situ detection of cyanobacteria based on matrix model}

\author{
LEI Chenglong ${ }^{1,2}$, JIANG Yuelu ${ }^{2}$, ZHOU $\mathrm{Jin}^{2}$, LAO Yongmin ${ }^{2}$, HE Yonghong ${ }^{2}$ \& CAI Zhonghua ${ }^{2 * *}$ \\ (1: School of Life Science, Tsinghua University, Beijing 100084, P.R.China) \\ (2: Graduate School at Shenzhen, Tsinghua University, Shenzhen 518055, P.R.China)
}

\begin{abstract}
Phycocyanin and chlorophyll-a are characterizations of cyanobacterial biomass. These two pigments are detected in situ with fluorescence spectrum method or fluorescence intensity. However, due to the fluorescence interference between phycocyanin and chlorophyll-a, the accuracy of in-situ detection of cyanobacteria is relatively low, which limited the application of this method. In this study, we developed a novel method of fluorescence analysis based on a two-order matrix model and a multivariate calibration linear model for the correlation between the fluorescence intensity and pigment concentration, which was verified by experiment. The established method decoupled the two mixed pigments as well as quantitatively analyzed the wavelength selection of excitation and emission and largely eliminated the fluorescence interference between phycocyanin and chlorophyll-a, and therefore, significantly improved the accuracy of in-situ detection of cyanobacteria. More importantly, our study can provide theoretical basis for the cyanobacteria detection sensor in how to select wavelengths.
\end{abstract}

Keywords: Phycocyanin; chlorophyll-a; fluorescence detection; decoupling; anti-interference

中国是世界上蓝藻水华暴发最严重、分布最广泛且蓝藻种类最多的国家之一 ${ }^{[1]}$, 太湖、滇池、巢湖等内 陆湖泊均已成为我国蓝藻水华暴发的高频地区 ${ }^{[2]}$. 蓝藻水华的暴发会引起水体缺氧而导致水生动植物大量 死亡, 破坏水体生态系统 ${ }^{[-4]}$; 其分泌的藻毒素进人水体后, 通过食物链、食物网或者饮用水进人人体, 对公 众健康造成极大的威胁 ${ }^{[5-6]}$; 藻类大量繁殖影响水质以及水厂的运行, 造成供水危机 ${ }^{[7]}$; 水华消亡时还会散 发恶臭气味, 影响周边的环境 ${ }^{[8]}$. 因此, 对蓝藻水华的预报、预警以及控制是水生生态环境治理的关键环节, 对蓝藻生物量的原位检测是蓝藻水华治理过程的核心, 是水华预报与预警的基础 ${ }^{[9]}$.

* 广东省海洋与渔业厅科技攻关与研发项目 (A201503D07) 和深圳市科技创新委计划项目 ( JCYJ20160608165926763, JCYJ20151117173236192, CXZZ20150529165045063) 联合资助. 2017 - 04 - 08 收稿; 2017-05-17 收修改稿. 类成龙 (1992 ), 男,硕士研究生; E-mail : chenglongl@ 139.com.

** 通信作者; E-mail: caizh@ sz.tsinghua.edu.cn. 
目前针对蓝藻生物量的检测主要有以下几种方法: 群落计数法 ${ }^{[10]}$ 、吸光光度法 ${ }^{[11-12]}$ 、多光谱遥感技 术 $^{[13]}$ 、苂光检测法 ${ }^{[14-17]}$ 、流式细胞法 ${ }^{[18]}$ 以及液相色谱法 ${ }^{[19]}$ 等. 这些方法给日常监测与科学研究带来便利的 同时,也均存在不足, 主要体现在精度、准确性、时效性以及人耗/时耗等方面, 尤其在原位监测上, 更是存在 瓶颈 ${ }^{[20]}$. 蓝藻生物量的菼光检测法因其简便性、灵敏度高、可原位检测等特点而被广泛使用, 其中多以藻蓝 蛋白作为特征色素, 利用二维苂光光谱法进行检测 ${ }^{[21]}$. 目前用苂光检测法检测蓝藻生物量的大多数研究皆 是在藻蓝蛋白的激发光谱与发射光谱中选择激发光与发射光波长 (多选择激发光谱与发射光谱的峰值波 长), 并建立藻蓝蛋白浓度与苂光强度的线性关系 ${ }^{[15,17,22-27]}$. 这些研究方法在单一色素的检测中表现出很高 的精确度, 而在实际水体中, 因藻蓝蛋白与叶绿素 a 苂光效应的相互干扰而影响测量精度, 从而难以将蓝藻 与其他真核藻类进行有效区分,极大限制了该方法的原位应用.

本文以简单易行的苂光分析法为基础, 运用二阶矩阵模型, 建立了一种以减少两种色素相互干扰为目 标的特定波长篮选方法,再结合多元线性回归分析对藻蓝蛋白、叶绿素 $\mathrm{a} 、$ 混合藻类的检测实验进行验证. 验 证结果表明, 本文方法可抑制藻蓝蛋白与叶绿素 $\mathrm{a}$ 的相互耦合, 避免其他真核藻类对蓝藻检测的干扰, 使藻 蓝蛋白的检测达到抗苂光干扰的效果, 从而实现蓝藻生物量的精确检测.

\section{1 材料与方法}

\section{1 材料与仪器}

叶绿素 a 与藻蓝蛋白的标准品均为 Sigma 标准试剂 (藻蓝蛋白标准品: Sigma-52468; 叶绿素 a 标准品: Sigma-C5753), 所用溶剂为 $15 \%$ 的乙醇 PBS 溶液 (由 PBS 溶液中加人 $150 \mathrm{ml}$ 乙醇后定容至 $1 \mathrm{~L}$ 而得, 且在此 溶剂中, 叶绿素 a 与藻蓝蛋白可以实现较大范围浓度比的混合); 藻类培养使用 BG-11 培养基; 藻类计数采 用血球计数板; 实验中所用的仪器为苂光分光光度计 (TECON-INFINITE-M200PRO, 奥地利), 仪器参数为:

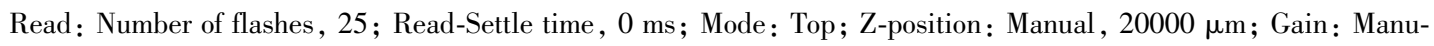
al , 110; Integration: time, $20 \mu \mathrm{s}$; 实验中所用的藻类包括铜绿微囊藻 (Microcystis aeruginosa) (蓝藻) 和小球藻 (Chlorella vulgaris, FACHB-8) (绿藻), 均来自中国科学院水生生物研究所淡水藻种库 (FACHB).

\section{2 波长篮选方法的建立}

蓝藻生物量的苂光检测法基于朗伯比尔定律 ${ }^{[25]}$, 以藻蓝蛋白为特征色素建立其浓度与苂光强度的关 系. 强度为 $I_{0}$ 的人射光平行照射到厚度为 $l$ 、浓度为 $c$ 的溶液表面, 当蓝藻溶液极稀 (满足 $K \cdot l \cdot c \leqslant 0.05$ ) 时 ${ }^{[14]}$, 荧光发射光强 $I_{\mathrm{f}}$ 为:

$$
I_{\mathrm{f}}=2.3 \eta \cdot I_{0} \cdot K \cdot l \cdot c
$$

式中, $\eta$ 为物质的苂光系数, $K$ 为物质的摩尔吸收系数.

在混合色素的苂光检测过程中, 不耦合检测是指检测过程中两种色素产生的苂光互不干扰. 而用苂光 分析法检测藻蓝蛋白与叶绿素 $\mathrm{a}$ 时, 两者存在很强的耦合效应. 结合多元线性回归分析以及苂光光谱的叠 加效应 ${ }^{[28]}$, 可建立多元线性苂光检测模型:

$$
\begin{aligned}
& u_{1}=h_{11} \cdot C_{1}+h_{12} \cdot C_{2}+b_{1} \\
& u_{2}=h_{21} \cdot C_{1}+h_{22} \cdot C_{2}+b_{2}
\end{aligned}
$$

式中, $u_{1}$ 和 $u_{2}$ 分别为叶绿素 $\mathrm{a}$ 和藻蓝蛋白的浓度; $h_{i j}$ 为线性系数; $C_{1}$ 和 $C_{2}$ 分别为叶绿素 $\mathrm{a}$ 和藻蓝蛋白检测方 法下的相对苂光强度值; $b_{1}$ 和 $b_{2}$ 分别为叶绿素 $\mathrm{a}$ 和藻蓝蛋白检测模型公式中的常数项, 即为朗伯比尔定律中 提出线性项后的残余项,也是仪器与环境噪声的反映项.

定义苂光系数矩阵 $(H)$ 为:

$$
H=\left(\begin{array}{ll}
h_{11} & h_{12} \\
h_{21} & h_{22}
\end{array}\right)
$$

由上,特定波长的不同组合会导致苂光系数矩阵中的元素不同, $H$ 阵也代表了不同色素之间的干扰程 度. 基于以上原理, 可以结合矩阵中向量相关性原理寻找两种色素相互干扰最小时的条件.

将 (2) 式转换为: 


$$
\begin{aligned}
& C_{1}=a_{11} \cdot u_{1}+a_{12} \cdot u_{2}+m_{1} \\
& C_{2}=a_{21} \cdot u_{1}+a_{22} \cdot u_{2}+m_{2}
\end{aligned}
$$

定义检测矩阵 $(A)$ 为:

$$
A=\left(\begin{array}{ll}
a_{11} & a_{12} \\
a_{21} & a_{22}
\end{array}\right)
$$

在不耦合双色素系统的检测中,菼光强度与色素浓度关系的模型应为:

$$
\begin{aligned}
& C_{1}=a_{11} \cdot u_{1}+m_{1} \\
& C_{2}=a_{22} \cdot u_{2}+m_{2}
\end{aligned}
$$

也即 $A$ 阵应为对角阵. 因而对于不同的方法组合, 需要寻找一种方法使得检测矩阵最接近于对角阵, 也 即使得 $A$ 阵的两个行向量相关性最差.

定义检测解耦参数 $(S)$ 为:

$$
S=|A|=a_{11} \cdot a_{22}-a_{12} \cdot a_{22}
$$

则由向量相关性理论可推导, $S$ 值越大, 则矩阵中两个行向量相关性越小. 因此 $S$ 值可表示荧光检测叶 绿素 $\mathrm{a}$ 与藻蓝蛋白时两者菼光光谱实现分离的程度,也即分离程度越大,两者的相互干扰越小.

通过对激发波长与发射波长的不同组合, 得到一系列 $A$ 矩阵, 找到 $S$ 值最大时所对应的 $A$ 矩阵, 此时的 方法组合即为最优的激发光波长与发射光波长. 而后根据多元线性回归分析建立苂光强度与两种色素浓度 的关系,即为检测藻蓝蛋白与叶绿素 $\mathrm{a}$ 混合色素的模型.

具体激发光波长与发射光波长篮选流程如图 1 所示. 其中, $C_{1} 、 C_{2}$ 分别为预选的检测叶绿素 $\mathrm{a}$ 的激发光 波长范围与发射光范围; $P_{1} 、 P_{2}$ 分别为预选的检测藻蓝蛋白的激发光波长范围与发射光范围; $N_{i}$ 为特定波长 组合的方法 $i$. 所有数据处理在 Matlab 软件(Matlab-R2012a) 中完成, 实现 $S$ 值的篮选.

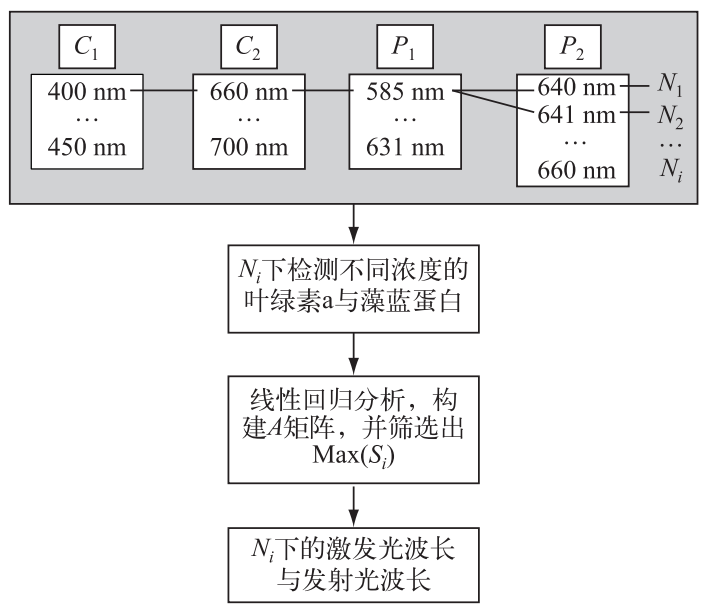

图 1 波长篮选方法

Fig.1 Method of wavelength selection

\section{3 验证实验}

在验证实验中, 对以下 3 种方法做对比:

本文研究方法 (M1) : 是指以本研究所篮选出的激发光波长与发射光波长进行检测, 结合多元线性回归 分析建立检测模型;

最强波长模型 (M2): 是指以色素菼光光谱中的峰值波长进行检测, 建立模型方法同 $\mathrm{M}^{[28]}$;

标准曲线法 (M3) : 是指波长选择同 M2, 用单一色素的标准曲线法建立模型 ${ }^{[17,22-23]}$.

1.3.1 色素检测 将两种色素配制不同浓度梯度的混合溶液, 且保持每份溶液中叶绿素 a 与藻蓝蛋白浓度比 
$C_{\mathrm{Chl.a}}: C_{\mathrm{PC}}=1: 1$. 保持其他实验条件与前述相同, 用 $\mathrm{M} 1 、 \mathrm{M} 2 、 \mathrm{M} 33$ 种方法分别检测相应的苂光强度, 同样的浓 度取 3 个样本, 同样的样本连续测 3 次.

1.3.2 藻类的检测 本文用混合培养对数生长期的铜绿微囊藻与小球藻进行实验, 以藻蓝蛋白作为特征色素 检测铜绿微囊藻细胞浓度 $(\mathrm{cells} / \mathrm{ml})$ 与藻蓝蛋白苂光强度的关系. 通过对单一铜绿微囊藻的个数与藻蓝蛋 白浓度的线性关系, 建立蓝藻细胞浓度的检测模型. 藻液的培养条件为: $300 \mathrm{ml}$ 培养瓶, 藻液容积为 $200 \mathrm{ml}$; 温度为 $25 \pm 1^{\circ} \mathrm{C}$; 光照时间 $\mathrm{L}: \mathrm{D}=12 \mathrm{~h}: 12 \mathrm{~h}$, 光照强度为 $3300 \pm 200 \mathrm{~lx}$; 每天摇晃 1 次, 并随机交换位置以保证 每个培养瓶均接受到相同的光照; 培养周期为 $15 \mathrm{~d}$. 每天用 M1、M2、M3 3 种方法检测混合藻液中藻蓝蛋白 的苂光强度, 并计算蓝藻的细胞浓度. 蓝藻浓度标准值测量依据国家标准《水和废水监测分析方法》中的群 落计数法, 在显微镜下检测 ${ }^{[10]}$.

1.3.3 结果指标比较 在对色素标准品与活体藻类检测中, 用 3 个指标对结果进行评估: 准确率 (或者误 差)、精密度和回收率. 准确率的计算公式为:

$$
\begin{gathered}
\text { 准确率 }=\frac{\text { 检测值 }}{\text { 真值 }} \times 100 \% \\
\text { 误差 }(e)=1-\text { 准确率 }
\end{gathered}
$$

式中, 检测值为检测结果; 真值在色素检测实验中为色素配比时的标准浓度值, 在活体藻类检测中则指显微 计数法所获得的浓度值.

标准差表征多次检测值的可重复性, 也即为检测结果的稳定性; 其值越小, 表示精密度越高. 在本文实 验中,精密度为同一浓度样品 6 次测量结果的标准差.

回收率用来表征检测方法的准确度高低,其计算公式为：

$$
\text { 回收率 }=\frac{\text { 加标后混合样品的平均测量值 }- \text { 加标前样品的平均测量值 }}{\text { 加标量 }} \times 100 \%
$$

式中,加标量为加标前浓度的 $20 \%$ 且在检测范围内.

\section{2 结果与讨论}

\section{1 叶绿素 a 与藻蓝蛋白的荧光光谱特征}

激发光谱中 (选取叶绿素 $\mathrm{a}$ 的发射波长为 $680 \mathrm{~nm}$, 藻蓝蛋白的发射波长为 $648 \mathrm{~nm}$ ), 叶绿素 $\mathrm{a}$ 的峰值出 现在 $436 \mathrm{~nm}$, 而且从 $500 \mathrm{~nm}$ 到 $630 \mathrm{~nm}$ 逐渐升高, 藻蓝蛋白的峰值出现在 $626 \mathrm{~nm}$, 虚线框内两者具有很强的 重叠耦合 (图 2a) ; 发射光谱中 (选取叶绿素 $\mathrm{a}$ 的激发波长为 $436 \mathrm{~nm}$, 藻蓝蛋白的激发波长为 $626 \mathrm{~nm}$ ), 叶绿 素 $\mathrm{a}$ 的峰值出现在 $680 \mathrm{~nm}$, 藻蓝蛋白的峰值出现在 $648 \mathrm{~nm}$, 虚线框内两者具有很强的重叠耦合 (图 2b). 当 蓝藻水华暴发时,水体中含有大量这两种色素, 彼此间的耦合则产生较强的苂光干扰.

当 $C_{\mathrm{Chl.a}}>500 \mu \mathrm{g} / \mathrm{L}$ 时, 叶绿素 $\mathrm{a}$ 的苂光强度与浓度不呈显著的线性关系; 当 $C_{\mathrm{PC}}>2000 \mu \mathrm{g} / \mathrm{L}$ 时, 藻蓝蛋 白的苂光强度与浓度也不呈显著的线性关系 (图 2c). 从曲线形状看两者更接近于幂函数曲线, 这与将朗伯 比尔定律进行泰勒级数分解后的结果一致: 此时不满足条件 $K \cdot l \cdot c \leqslant 0.05$, 则呈现一种幂函数关系 ${ }^{[14]}$. 但 此高浓度在实际水体检测中不常见, 因此本文只研究浓度在线性范围内的部分. 因而, 叶绿素 $\mathrm{a}$ 的苂光线性 浓度范围上限为 $500 \mu \mathrm{g} / \mathrm{L}$. 藻蓝蛋白的苂光线性浓度范围上限为 $2000 \mu \mathrm{g} / \mathrm{L}$. 将图 2c 中浓度范围为 $0.03 \sim$ $500 \mu \mathrm{g} / \mathrm{L}$ 的部分放大至图 $2 \mathrm{~d}$, 可知当 $C_{\mathrm{Chl.a}}>0.10 \mu \mathrm{g} / \mathrm{L}$ 或者 $C_{\mathrm{PC}}>0.20 \mu \mathrm{g} / \mathrm{L}$ 时, 色素苂光强度与浓度呈现出 很强的线性关系. 其中叶绿素 $\mathrm{a}$ 的相关系数 $R^{2}$ 达 0.9944 , 藻蓝蛋白的相关系数 $R^{2}$ 达 0.9942 . 浓度低于这个范 围时, 检测已经无区分度. 因此, 本文所研究的叶绿素 a 和藻蓝蛋白的苂光线性浓度范围分别为 $0.10 \sim 500$ $\mu \mathrm{g} / \mathrm{L}$ 和 $0.20 \sim 2000 \mu \mathrm{g} / \mathrm{L}$. 藻蓝蛋白的线性浓度范围更广, 适合在高浓度下检测, 满足水华暴发时的测量需 求; 而叶绿素 $\mathrm{a}$ 的检测限较低, 灵敏度高, 但是检测范围相比藻蓝蛋白小.

\section{2 叶绿素 $\mathrm{a}$ 与藻蓝蛋白干扰程度最小波长的䇻选}

按照第 1 节中的方法收集叶绿素 a 与藻蓝蛋白的苂光光谱数据, 在 Matlab 软件 (Matlab-R2012a) 中运行 本文提出的波长篎选方法模型, 最终得出可最大程度上减弱两种色素之间荧光干扰的波长组合: 叶绿素 $\mathrm{a}$ 和藻蓝蛋白的激发波长分别为 440 和 $600 \mathrm{~nm}$, 发射波长分别为 688 和 $640 \mathrm{~nm}$. 

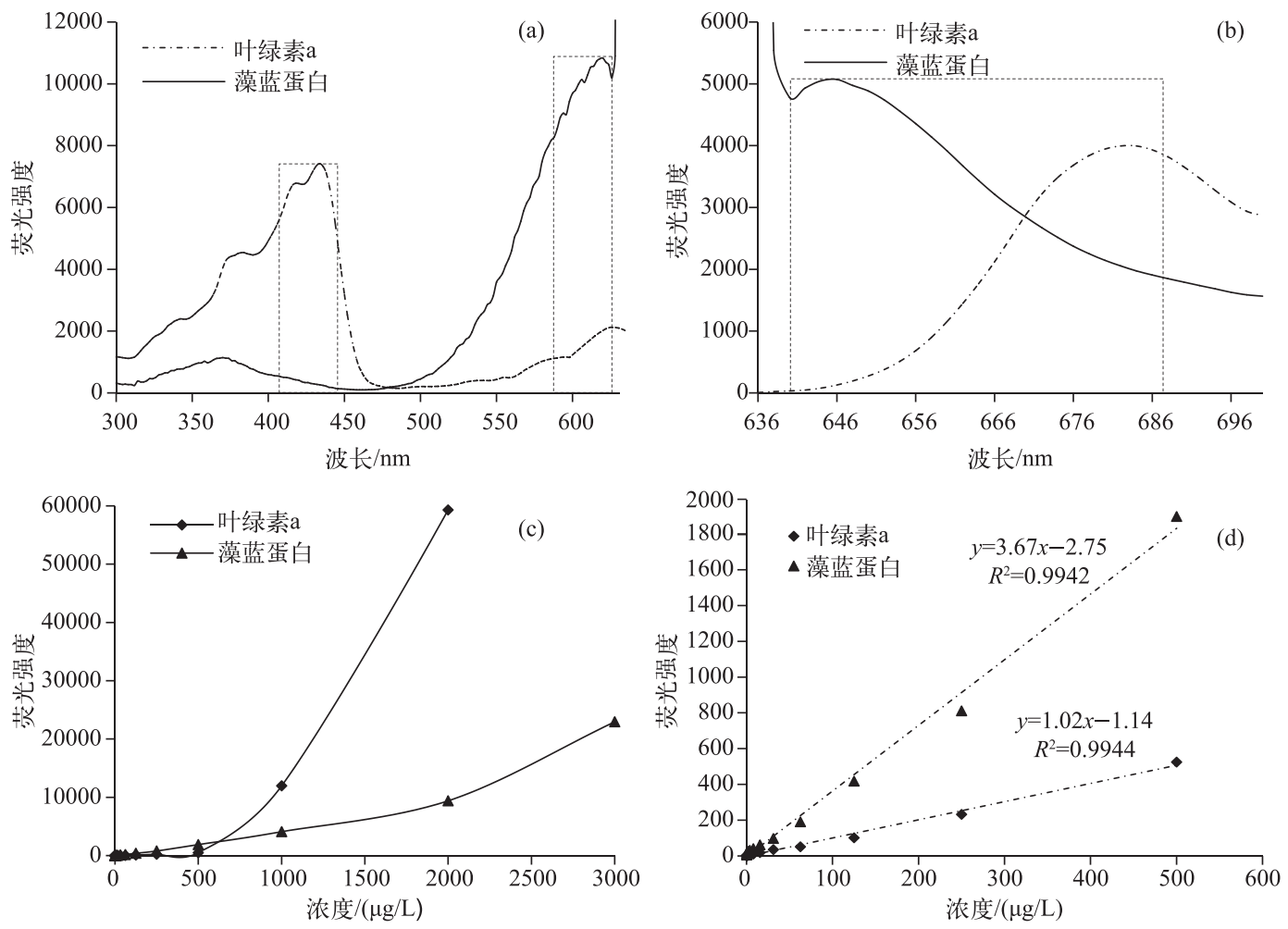

图 2 叶绿素 $\mathrm{a}$ 与藻蓝蛋白的苂光光谱性质 (叶绿素 $\mathrm{a}$ 与藻蓝蛋白的激发光谱 (a);

叶绿素 a 与藻蓝蛋白的发射光谱 (b) ; 叶绿素 a 与藻蓝蛋白的苂光强度-浓度相关关系曲线 (c) ; 叶绿素 a 与藻蓝蛋白在低浓度下的苂光强度-浓度相关关系曲线 (d) )

Fig.2 Excitation spectrum and emission spectrum of chlorophyll-a and phycocyanin

\section{3 叶绿素 a 与藻蓝蛋白的浓度检测}

在叶绿素 a 与藻蓝蛋白混合色素溶液的检测中 (图 3), 本文研究方法 M1 得到叶绿素 a 和藻蓝蛋白的 灵敏度分别为 0.06 和 $0.1 \mu \mathrm{g} / \mathrm{L}$, 相比于方法 M2 与 M3 的结果具有一定提高. 3 种方法的检测上限并无明显 差异, 与图 2c 所显示的一致, 叶绿素 a 检测上限为 $500 \mu \mathrm{g} / \mathrm{L}$, 藻蓝蛋白检测上限为 $2000 \mu \mathrm{g} / \mathrm{L} . \mathrm{M} 1$ 方法中曲 线线性程度最好, 相关系数 $R^{2}$ 分别达到 0.9948 与 0.9936 , 比 M2 方法和目前研究中常用的 M3 方法有显著提 高. 这说明本文研究方法能够有效提高混合色素中叶绿素 a 与藻蓝蛋白检测的灵敏度与检测稳定性,提高 检测精度.

而无论是检测叶绿素 a 还是藻蓝蛋白,相同的色素浓度在 3 种方法检测下, 菼光强度呈现的规律均为: $\mathrm{M} 1<\mathrm{M} 2<\mathrm{M} 3$. 原因在于 $\mathrm{M} 2$ 方法使用光谱中的峰值波长进行激发, 所得到的发射光会略强于非峰值波长的激 发结果, 且在峰值波长作为激发光与发射光波长时,色素之间的苂光干扰也比 M1 方法要大. 而 M3 中相同 浓度色素的苂光强度最大, 则主要是因为色素之间的干扰. 因此, 不同的波长组合对检测结果具有一定影 响, 使用光谱中的峰值波长作为检测波长, 苂光强度固然增大, 但拟合效果未必是最佳. 相反, 本文研究方法 则具有更高的灵敏度与更低的检测下限, 且线性程度更优. 如此, 可提高检测的稳定性, 使藻蓝蛋白的检测 效果更好.

取 $1 、 10$ 和 $100 \mu \mathrm{g} / \mathrm{L} 3$ 种浓度的混合色素( 保持每份溶液中 $C_{\mathrm{Chla}}: C_{\mathrm{PC}}=1: 1$ ), 用 1.3 .1 节中的描述方法 进行检测 (图 4). 比较 M1 与 M2 方法的结果 (图 4a), 可发现在两种色素的线性浓度范围内, M1 所得到的结 果优于 M2, 说明不同特定波长的选择对藻蓝蛋白与叶绿素 $\mathrm{a}$ 的检测精度产生影响, 而不是单一色素检测下 苂光强度越强越好. M2 方法劣于 M1, 一方面是因为选择光谱中最强的峰值波长而未考虑两种色素之间的 


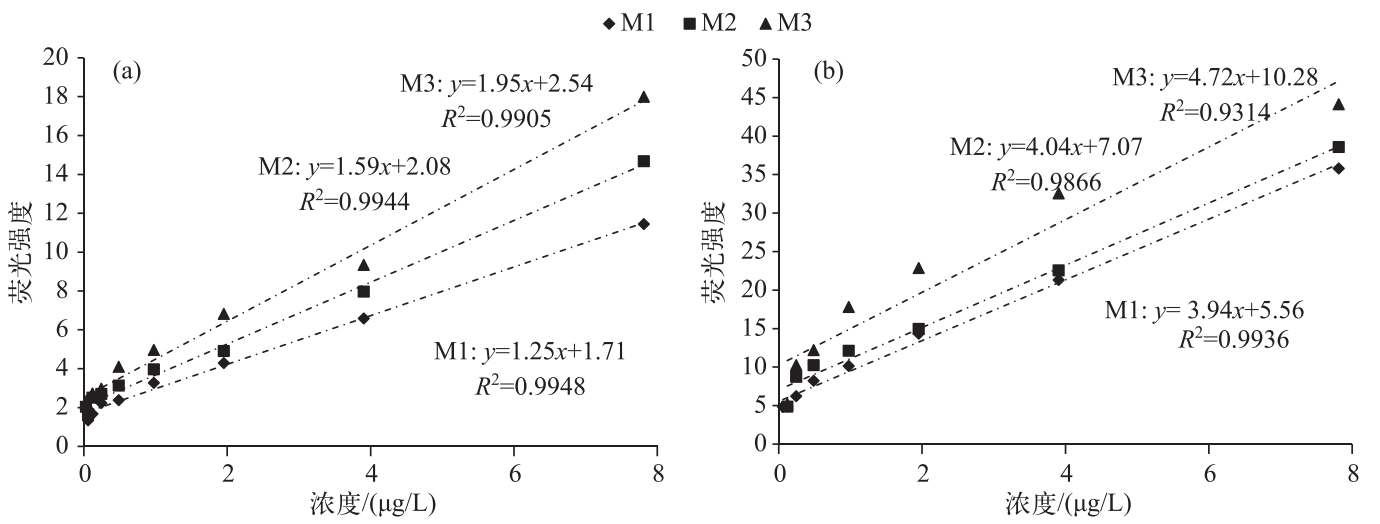

图 3 叶绿素 $\mathrm{a}(\mathrm{a})$ 和藻蓝蛋白 (b) 在 3 种方法检测下的线性拟合结果

Fig.3 Linear correlation of chlorophyll-a (a) and phycocyanin (b) in fluorescence detection with the three methods

耦合影响; 另一方面则是因为菼光强度大,放大了因苂光不稳定所带来的误差效应. 比较 M2 与 M3 方法的 结果, 发现 M2 方法明显优于 M3 方法, 说明多元校正线性模型对于提高两种色素的检测精确度同样具有很 大影响. 直观从检测结果来看, 本文研究方法通过笁选苂光检测中的激发光波长与发射光波长, 能显著提高 蓝藻检测精度. 相比于目前普遍使用的 M3 方法, 对波长进行笁选并建立多元线性回归模型具有显著优势 ( One-way ANOVA, $F>F$ crit, $P$-value $<0.05$ ).

对 3 种检测方法下的结果进行定量分析, 比较准确率、精密度与回收率 3 个指标 (皆为平均值) (图 4b), 发现 M1 方法检测叶绿素 a 与藻蓝蛋白的准确率分别为 $90.4 \%$ 和 $92.0 \%$, 相比于 M2 和 M3 方法分别高 出 $2.0 \% \sim 20.0 \%$ 和 $4.3 \% \sim 20.0 \%$, 具有明显的优势; 从精密度来看, M1 方法的平均标准差最小, 表征精密度 最高, 说明本研究方法的精密度与重复性更好, 避开最强波长的苂光, 在一定程度上可以减弱苂光不稳定性 所带来的误差, 实现了两种色素抗干扰性最强, 提高了检测结果的精密度; 从回收率来看, M1 方法的准确性 最高, 更加验证了本研究结果在检测效果上的优势. 综合上述参数的比较, 本研究方法能提高混合色素检测 的准确率,有效减弱两种色素间的耦合作用,并具有较好的稳定性.
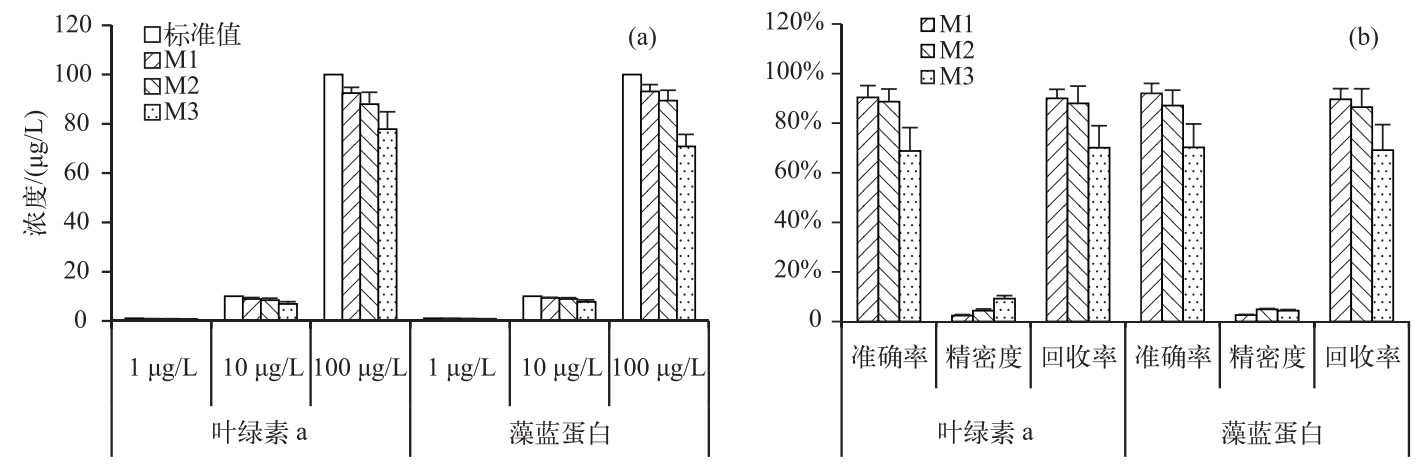

图 43 种方法下叶绿素 a 与藻蓝蛋白不同混合浓度的检测结果:

3 种方法与标准浓度的比较 (a) ; 3 种方法检测指标的比较 (b)

Fig.4 Detecting the concentration of chlorophyll-a and phycocyanin mixed with the three methods

\section{4 蓝藻与真核藻类混合浓度的检测}

藻蓝蛋白作为蓝藻的特征色素, 其浓度与蓝藻数量呈现高度的相关性 ${ }^{[26]}$. 在 BG-11 培养基中进行铜绿 微囊藻与小球藻的混合培养, 通过上述 3 种验证方法对对数生长期内藻蓝蛋白的菼光强度与蓝藻细胞浓度 
进行线性回归分析.

3 种方法中, M1 方法下线性回归方程为: $y=0.94 x-0.29, R^{2}=0.9994$, 其斜率最接近于 1 , 且相关系数最 大 (图 5a). M2 方法的检测结果与 M1 较为接近, 但依然不如 M1 检测效果好, 且 M1 与另外两种方法的检测 结果具有显著性差异 (One-way ANOVA, $F>F$ crit, $P$-value $<0.05$ ), 从图 $5 \mathrm{~b}$ 可直观看出 3 种方法检测效果的 差异. 以显微计数值作为模拟值计算 Nash-Sutcliffe 效率系数为 0.94 , 相比于陈纬栋等 ${ }^{[25]}$ 的 0.78 以及 Gregor 等 ${ }^{[29]}$ 的 1.12 具有较大改进; 相关系数高于杨飞等 ${ }^{[17]}$ 的研究, 检测结果更加稳定; 准确率方面比 Lee 等 ${ }^{[15]}$ 的 $80 \%$ 90\% 也有较大提高.
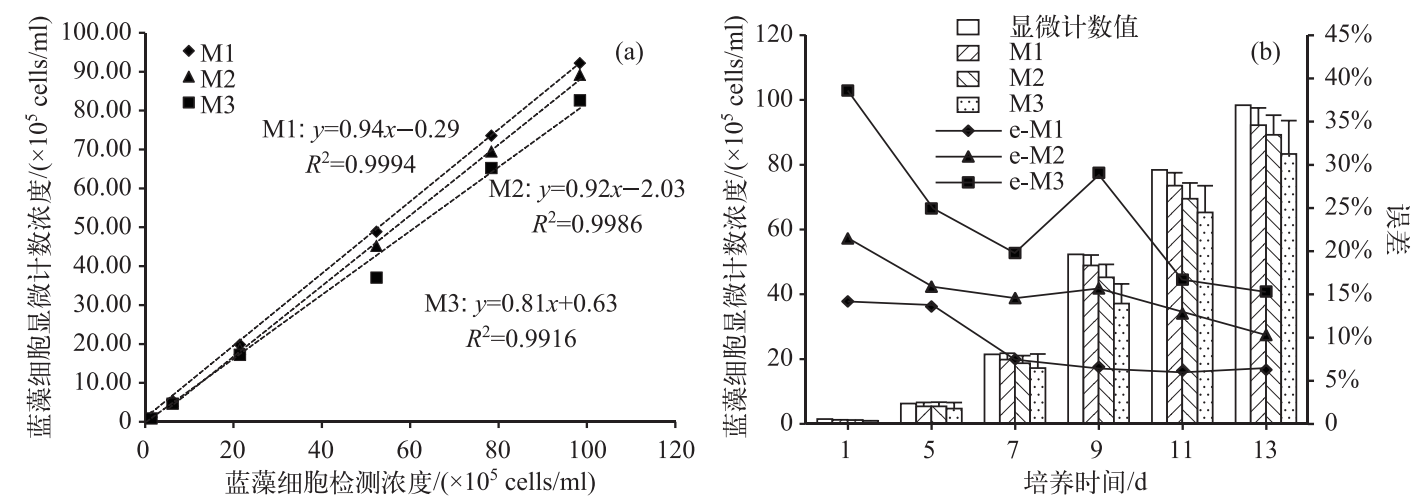

图 5 混合藻类在 3 种方法检测下的结果 (e-M1、e-M2、e-M3 分别为 M1、M2、M3 方法的检测误差)

Fig.5 Detecting results of Microcystis aeruginosa and Chlorella vulgaris mixing solution with the three methods

比较图 5 与图 4 发现, 在实际检测活体藻类的过程中, 准确率相比于色素的混合检测略有下降. 其主要 是因为对游离的藻蓝蛋白的测定与细胞中原始状态不同, 不同种类蓝藻、同种蓝藻的不同细胞内光合色素 系统的结构差异以及相同细胞在不同生长环境与不同阶段中的差异也会对苂光结果产生影响 ${ }^{[15]}$. 本研究 将蓝藻光合系统中色素之间复杂的相互作用共同看作一个 “黑匣子”, 将其内部的差异看作系统误差来处 理,活体藻类色素系统的光合效应与标准色素相似, 从而可以看出本文研究方法在提高检测效果方面的优 势. 尽管本文研究方法的结果与混合藻类中蓝藻浓度的标准结果仍然呈现较好的相关关系, 但对其内部光 合色素系统的研究可为进一步提高蓝藻的检测精度提供很好的研究方向.

在混合藻类的培养过程中, 3 种方法的检测误差随着时间增加呈现下降趋势. 本文推测其原因为: 在 BG-11 “富营养” 水平下, 培养的开始阶段, 两种藻类的生长相互干扰较小, 环境提供足够的生长繁殖条件, 蓝 藻与绿藻都呈 “ $\mathrm{J}$ ” 型曲线增长, 且数量相当, 这与本文显微镜计数结果一致. 此时 $C_{\mathrm{Chl.a}}: C_{\mathrm{PC}}$ 较高, 叶绿素 $\mathrm{a}$ 与 藻蓝蛋白之间的苂光干扰较大, 从而导致总体检测误差大. 此阶段中, M1 的误差明显比另外两种方法小, 本

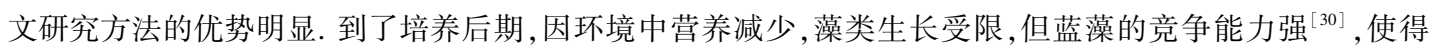
蓝藻浓度高于绿藻浓度, $C_{\mathrm{Chl.a}}: C_{\mathrm{PC}}$ 降低, 叶绿素 $\mathrm{a}$ 对藻蓝蛋白的苂光干扰降低, 从而导致总体检测误差变小. 总体而言, M1 的误差依然最小. 活体藻类的实验证明, 本文的研究方法能够显著提高混合藻类中蓝藻浓度 的检测精确度, 本研究结果同样适用于实际水体, 且在蓝藻占比越小时, 本研究方法越具优势.

\section{3 结论}

1) 使用本文所提出的二阶矩阵模型进行蓝藻检测过程中激发光波长与发射光波长的篮选, 可找出藻蓝 蛋白受叶绿素 a 干扰最小的波长组合. 所篎选的 “激发光一发射光”最优抗干扰波长组合结果为: 叶绿素 a 和 藻蓝蛋白的激发波长分别为 440 和 $600 \mathrm{~nm}$, 发射波长分别为 688 和 $640 \mathrm{~nm}$.

2) 对两种色素以及活体藻液的实验验证结果表明:使用本研究的波长篮选方法,结合多元线性回归分 析, 能够克服目前蓝藻检测研究中存在的波长选择问题, 抑制两种色素之间的耦合干扰, 提高检测的精准 度, 实现蓝藻生物量的抗苂光干扰原位检测. 
3 ) 本文研究方法在混合藻液中蓝藻占比越小时,抑制干扰提高检测精度的优势越明显.

4) 本研究为混合色素苂光检测提供了一种笁选抗干扰激发光波长与发射光波长的科学方法, 为藻蓝蛋 白苂光检测传感器的研制提供了理论基础,并具有业务化的潜力.

\section{4 参考文献}

[ 1 ] Yao Yuling, Jiang Wenjing, Qu Qiu et al. Progress toxin detection method of surface water and drinking water microcystin. Modern Scientific Instruments, 2008, (5): 86-90. [姚玉玲, 蒋文菁, 屈秋等. 地表水和饮用水中微囊藻毒素检测方 法的研究进展. 现代科学仪器, 2008, (5):86-90.]

[ 2 ] Shi Kun, Li Yunmei, Liu Zhonghua et al. Three wavelengths factor model of Chlorophyll concentrations inversion based on different spectral dominating factors for inland lake. Environmental Science, 2010, 31(12) : 2873-2881. [施坤, 李云梅, 刘忠华等. 基于不同光谱主导因子的内陆湖泊水体叶绿素浓度三波段反演模型研究. 环境科学, 2010, 31(12): 2873-2881. ]

[ 3 ] Otten TG, Paerl HW. Health effects of toxic cyanobacteria in U. S. Drinking and recreational waters: Our current understanding and proposed direction. Current Environmental Health Reports, 2015, 2(1):1-10.

[ 4 ] Falconer IR. Toxic cyanobacteria bloom problems in Australian waters: Risks and impacts on human health. Phycologia, 2001, 40(3) : 228-233.

[ 5 ] Xie Ping. A review on the studies related to the effects of microcystins on human health. J Lake Sci, 2009, 21( 5) : 603613. DOI: 10.18307/2009.0501. [谢平. 微囊藻毒素对人类健康影响相关研究的回顾. 湖泊科学, 2009, 21(5): 603-613. ]

[ 6 ] Machado J, Campos A, Vasconcelos V et al. Effects of microcystin-LR and cylindrospermopsin on plant-soil systems: A review of their relevance for agricultural plant quality and public health. Environmental Research, 2016, 153:191-204.

[ 7 ] Harrison PJ, Piontkovski S, Al-Hashmi K. Understanding how physical-biological coupling influences harmful algal blooms, low oxygen and fish kills in the Sea of Oman and the Western Arabian Sea. Marine Pollution Bulletin, 2016, 114 (1) : 25-34.

[ 8 ] Jüttner F, Watson SB. Biochemical and ecological control of geosmin and 2-methylisoborneol in source waters. Applied \& Environmental Microbiology, 2007, 73(14) : 4395-4406.

[ 9 ] Izydorczyk K, Tarczynska M, Jurczak T et al. Measurement of phycocyanin fluorescenceas an online early warning system for cyanobacteria in reservoir intake water. Environmental Toxicology, 2005, 20(4) : 425-430.

[10] “Water and wastewater monitoring and analysis technology" editorial board State in Environmental Protection Administration ed. Water and wastewater monitoring and analysis technology: Fourth Edition. Beijing: China Environmental Science Press, 2002. [ 国家环境保护总局《水和废水监测分析方法》编委会. 水和废水监测分析方法: 第四版. 北京: 中国 环境科学出版社, 2002.]

[11] Ahn CY, Joung SH, Yoon SK et al. Alternative alert system for cyanobacterial bloom, using phycocyanin as a level determinant. Journal of Microbiology, 2007, 45(2) :98-104.

[12] Bennett A, Bogorad L. Complementary chromatic adaptation in a filamentous blue-green alga. Journal of Cell Biology, $1973, \mathbf{5 8}(2)$ : 419-435.

[13] Lunetta RS, Schaeffer BA, Stumpf RP et al. Evaluation of cyanobacteria cell count detection derived from MERIS imagery across the eastern USA. Remote Sensing of Environment, 2014, 157 : 24-34.

[14] Brechet E, Mcstay D, Wakefield RD et al. Novel blue LED-based handheld fluorometer for detection of terrestrial algae on solid surfaces. Proceedings of SPIE-The International Society for Optical Engineering, 1998, 3414:184-190.

[15] Lee TY, Tsuzuki M, Takeuchi T et al. Quantitative determination of cyanobacteria in mixed phytoplankton assemblages by an in vivo fluorimetric method. Analytica Chimica Acta, 1995, 302(1) : 81-87.

[16] Yentsch CS, Yentsch CM. Fluorescence spectral signatures: the characterization of phytoplankton populations by the use of excitation and emission spectra [Algae, diatoms]. Applied \& Environmental Microbiology, 1979, 60( 8) : 2911-2915.

[17] Yang Fei, Tao Zhihao, Zheng Guilin. Design of a long-term and real-time in-situ monitor for cyanobacterial biomass. Chinese Journal of Sensors and Actuators, 2016, 29(5) : 769-775. [ 杨飞, 陶志浩, 郑贵林. 一种蓝藻生物量长期原位实 时监测仪的研制. 传感技术学报, 2016, 29(5): 769-775.] 
[18] Xu Zhaoan, Gao Yi, Wu Donghao. Application of flow cytometry in monitoring algae in Taihu. Environmental Monitoring in China, 2012, 28 (4) : 69-73. [徐兆安, 高怡, 吴东浩等. 应用流式细胞仪监测太湖藻类初探. 中国环境监测, $2012, \mathbf{2 8}(4): 69-73$.

[19] Sørensen LK, Snor LK. Determination of eight penicillins in serum from cattle and pigs by generic HPLC method. Chromatographia, 2001, 53(7): 367-371.

[20] Gao Yahui, Yang Junxia, Luo Qiaoqi et al. Automatic identification and analysis techniques of marine phytoplankton. Journal of Xiamen University: Natural Science, 2006, (S2) : 40-45. [高亚辉, 杨军霞, 骆巧琦等. 海洋浮游植物自动分析 和识别技术. 厦门大学学报: 自然科学版, 2006, (S2) : 40-45.]

[21] Jin HL, Wang YT. Hybrid alga identify method research based on fluorescence and analysis of multi-element regression analysis. Chinese Journal of Sensors \& Actuators, 2006, 19(4) : 1198-1201.

[22] Silva T, Giani A, Figueredo C et al. Comparison of cyanobacteria monitoring methods in a tropical reservoir by in vivo, and in situ, spectrofluorometry. Ecological Engineering, 2016, 97: 79-87.

[23] Beutler M, Wiltshire KH, Arp M et al. A reduced model of the fluorescence from the cyanobacterial photosynthetic apparatus designed for the in situ detection of cyanobacteria. Biochimica et Biophysica Acta, 2003, 1604(1): 33 .

[24] Wang Jinxia, Luo Guyuan. Detecting cyanobacterial biomass by fluorescence spectrometry. Modern Scientific Instruments, 2011, (6)：111-113. [王金霞, 罗固源. 应用苂光光谱法检测蓝藻生物量. 现代科学仪器, 2011, (6)：111113. ]

[25] Chen Weidong, Wang Chong, Hu Xiaofang et al. Application of fluorescent analysis technology for cyanobacterial biomass detection. Water Purification Technology, 2010, 29(6) : 80-84. [陈纬栋, 王崇, 胡晓芳等. 应用苂光分析技术检测蓝 藻生物量. 净水技术, 2010, 29(6): 80-84.]

[26] Yang Dingtian, Pang Delu. Optical detection of phycoeyanin in waters. Chinese Journal of Scientific Instrument, 2006, 27 (S2) : 1033-1035. [杨顶田, 潘德炉. 水体中藻蓝蛋白的光谱检测. 仪器仪表学报, 2006, 27(S2) : 1033-1035.]

[27] Jin Hailong, Wang Yutian. Hybrid Alga identify method research based on fluorescence and analysis of multi-element regression analysis. Chinese Journal of Sensors and Actuators, 2006, 19(1) : 97-99. [金海龙, 王玉田. 基于苂光发射光 谱的活体海藻识别方法研究. 传感技术学报, 2006, 19(1):97-99.]

[28] Yu Xiaoya, Zhang Yujun, Yin Gaofang. Feature wavelength selection of phytoplankton fluorescence spectra based on partial least squares. Acta Optica Sinica, 2014, 34(9): 294-299. [余晓娅, 张玉钧, 殷高方等. 基于偏最小二乘回归的 藻类苂光光谱特征波长选取. 光学学报, 2014, 34(9) : 294-299.]

[29] Gregor J, Marsálek B, Sípková H. Detection and estimation of potentially toxic cyanobacteria in raw water at the drinking water treatment plant by in vivo fluorescence method. Water Research, 2007, 41(1) : 228.

[30] Alstyne KLV, Nelson TA, Ridgway RL. Environmental chemistry and chemical ecology of “Green Tide” seaweed blooms. Integrative \& Comparative Biology, 2015, 55(3) : 518. 